\title{
Immunofluorescence characterization of spinal cord dorsal horn microglia and astrocytes in horses
}

Constanza Stefania Meneses ${ }^{1}$, Heine Yacob Müller ${ }^{1}$, Daniel Eduardo Herzberg ${ }^{1}$, Benjamín Uberti ${ }^{2}$, Hedie

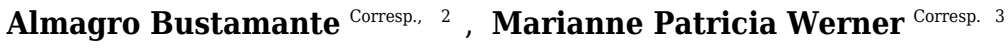

${ }^{1}$ Veterinary Sciences Graduate School, Universidad Austral de Chile, Valdivia, Chile

2 Veterinary Clinical Sciences Department, Universidad Austral de Chile, Valdivia, Chile

3 Animal Science Department, Universidad Austral de Chile, Valdivia, Chile

Corresponding Authors: Hedie Almagro Bustamante, Marianne Patricia Werner

Email address: hbustamante@uach.cl, marianne.werner@uach.cl

Role of glial cells in pain modulation has recently gathered attention. Objective of this study was to determine healthy spinal microglia and astrocyte morphology and disposition in equine spinal cord dorsal horns using Iba-1 and GFAP/CX-43 immunofluorescence labeling, respectively. Five adult horses without visible wounds or gait alterations were selected. Spinal cord segments were obtained post-mortem for immunohistochemical and immunocolocalization assays. Immunodetection of spinal cord dorsal horn astrocytes was done using a polyclonal goat antibody raised against Glial Fibrillary Acidic Protein (GFAP) and a polyclonal rabbit antibody against Connexin 43 (Cx-43). For immunodetection of spinal cord dorsal horn microglia, a polyclonal rabbit antibody against a synthetic peptide corresponding to the C-terminus of ionized calcium-binding adaptor molecule 1 (Iba-1) was used. Epifluorescence and confocal images were obtained for the morphological and organizational analysis. Evaluation of shape, area, cell diameter, cell process length and thickness was performed on dorsal horn microglia and astrocyte. Morphologically, an amoeboid o spherical shape with a mean cell area of $92.4+34 \mu \mathrm{m} 2$ (in lamina I, II and III) was found in horse microglial cells, located primarily in laminae I, II and III. Astrocyte primary stem branches (and cellular bodies to a much lesser extent) are mainly detected using GFAP. Thus, double GFAP/Cx-43 immunolabeling was needed in order to accurately characterize the morphology, dimension and cell density of astrocytes in horses. Horse and rodent astrocytes seem to have similar dimensions and localization. Horse astrocyte cells have an average diameter of $56+14 \mu \mathrm{m}$, with a main process length of $28+8 \mu \mathrm{m}$, and thickness of $1.4+0.3 \mu \mathrm{m}$, mainly situated in laminae I, II and III. Additionally, a close association between end-point astrocyte processes and microglial cell bodies was found. These results are the first characterization of cell morphology and organizational aspects of horse spinal glia. Iba-1 and GFAP/Cx-43 can successfully immune-label microglia and astrocytes respectively in horse spinal cords, and thus reveal cell morphology and 
corresponding distribution within the dorsal horn laminae of healthy horses. The conventional hyper-ramified shape that is normally visible in resting microglial cells was not found in horses. Instead, horse microglial cells had an amoeboid o spherical shape. Horse protoplasmic astroglia is significantly smaller and structurally less complex than human astrocytes, with fewer main GFAP processes. Instead, horse astrocytes tend to be similar to those found in rodent's model, with small somas and large cell processes. Microglia and astrocytes were found in the more superficial regions of the dorsal horn, similarly to that previously observed in humans and rodents. Further studies are needed to demonstrate the molecular mechanisms involved in the neuron-glia interaction in horses. 
1 IMMUNOFLUORESCENCE CHARACTERIZATION OF SPINAL CORD DORSAL

2 HORN MICROGLIA AND ASTROCYTES IN HORSES

3 Constanza Stefania Meneses ${ }^{3}$, Heine Yacob Müller ${ }^{3}$, Daniel Eduardo Herzberg ${ }^{3}$, Benjamín

4 Uberti $^{2}$, Hedie Almagro Bustamante ${ }^{2}$, Marianne Patricia Werner ${ }^{1}$

$5 \quad{ }^{1}$ Animal Science Department, Universidad Austral de Chile, Valdivia, Chile

$6 \quad{ }^{2}$ Veterinary Clinical Sciences Department, Universidad Austral de Chile, Valdivia, Chile

$7 \quad 3$ Veterinary Sciences Graduate School, Universidad Austral de Chile, Valdivia, Chile

8

9 Corresponding Author:

10 Marianne Werner ${ }^{1}$

11 Independencia 641, Valdivia, 5090000, Chile

12 Email address: marianne.werner@uach.cl 


\section{ABSTRACT}

24

25

26

27

28

29

30

31

32

33

34

35

36

3

38

41 Morphologically, an amoeboid o spherical shape with a mean cell area of $92.4 \pm 34 \mu \mathrm{m} 2$ (in

42 lamina I, II and III) was found in horse microglial cells, located primarily in laminae I, II and III.

43 Astrocyte primary stem branches (and cellular bodies to a much lesser extent) are mainly

Background

Role of glial cells in pain modulation has recently gathered attention. The objective of this study was to determine healthy spinal microglia and astrocyte morphology and disposition in equine spinal cord dorsal horns using Iba-1 and GFAP/Cx-43 immunofluorescence labeling, respectively.

\section{Methods}

Five adult horses without visible wounds or gait alterations were selected. Spinal cord segments were obtained post-mortem for immunohistochemical and immunocolocalization assays. Immunodetection of spinal cord dorsal horn astrocytes was done using a polyclonal goat antibody raised against Glial Fibrillary Acidic Protein (GFAP) and a polyclonal rabbit antibody against Connexin $43(\mathrm{Cx}-43)$. For immunodetection of spinal cord dorsal horn microglia, a polyclonal rabbit antibody against a synthetic peptide corresponding to the $\mathrm{C}$-terminus of ionized calcium-binding adaptor molecule 1 (Iba-1) was used. Epifluorescence and confocal images were obtained for the morphological and organizational analysis. Evaluation of shape, area, cell diameter, cell process length and thickness was performed on dorsal horn microglia and astrocyte.

\section{Results}

detected using GFAP. Thus, double GFAP/Cx-43 immunolabeling was needed in order to 
45 accurately characterize the morphology, dimension and cell density of astrocytes in horses.

46 Horse and rodent astrocytes seem to have similar dimensions and localization. Horse astrocyte

47 cells have an average diameter of $56 \pm 14 \mu \mathrm{m}$, with a main process length of $28 \pm 8 \mu \mathrm{m}$, and

48 thickness of $1.4 \pm 0.3 \mu \mathrm{m}$, mainly situated in laminae I, II and III. Additionally, a close

49 association between end-point astrocyte processes and microglial cell bodies was found.

50 Discussion

51 These results are the first characterization of cell morphology and organizational aspects of horse

52 spinal glia. Iba-1 and GFAP/Cx-43 can successfully immuno-label microglia and astrocytes

53 respectively in horse spinal cords, and thus reveal cell morphology and corresponding

54 distribution within the dorsal horn laminae of healthy horses. The conventional hyper-ramified

55 shape that is normally visible in resting microglial cells was not found in horses. Instead, horse

56 microglial cells had an amoeboid o spherical shape. Horse protoplasmic astroglia is significantly

57 smaller and structurally less complex than human astrocytes, with fewer main GFAP processes.

58 Instead, horse astrocytes tend to be similar to those found in rodent's model, with small somas

59 and large cell processes. Microglia and astrocytes were found in the more superficial regions of

60 the dorsal horn, similarly to that previously observed in humans and rodents. Further studies are

61 needed to demonstrate the molecular mechanisms involved in the neuron-glia interaction in

62 horses.

63

64

65 


\section{INTRODUCTION}

69

70

Glial cells have historically been considered as supporting cells for neuronal synapsis in the central nervous system (CNS), providing neurochemical precursors and energy sources, in addition to regulating the extracellular ionic concentration and removing cellular debris, among others (Haydon, 2001; Watkins et al., 2007). Interestingly, over the past two decades, the role of glial cells in pain modulation has gathered much attention (Gosselin et al., 2010). Scientific evidence has implicated microglia and astrocytes as key cells underlying acute pain and development of chronic pain after peripheral and central injury (Hains \& Waxman, 2006; Chen et al., 2012). Painful syndromes, like traumatic spinal cord injury (SCI), are associated with glial response that can dynamically modulate the function of CNS neurons, increasing their excitability and reactivity (Temburni \& Jakob, 2001). This differential functionality of glial cells is determined by the activation state of the cell and the components it expresses, which are different in duration of action and intensity according to the stimulus that triggers the glial reaction (Watkins et al., 2005). Glial-derived mediators powerfully modulate excitatory and inhibitory synaptic transmission at presynaptic, postsynaptic, and extrasynaptic sites (Ji et al., 2013), and moreover, are involved in the central modifications underlying chronic pain (Gosselin et al., 2010).

Glial activation is characterized by the upregulation of specific glial markers and/or morphological changes, including hypertrophy, proliferation, and modification of glial networks (Ji et al., 2013). After nerve damage, active microglia display morphological changes, such as switching from ramified to amoeboid shapes (Eriksson et al., 1993) and may show upregulation of the ionized calcium-binding adaptor molecule-1 (Iba-1), a small calcium binding protein 
90 specifically expressed by these cells (Hanisch \& Kettenmann, 2007). Several studies have 91 demonstrated that morphological changes associated to Iba-1 expression in correlate with cell

92 activation (Ito et al., 1998; Faustino et al., 2011). Furthermore, its overexpression is highly 93 correlated with increased nociceptive inputs converging to the dorsal spinal cord days after 94 injury (Yamamoto et al., 2015). Microglial activation seems to frequently occur during the early 95 phase of peripheral or central lesions and may even precede astrocyte changes (Tanga et al., 96 2004; Hald et al., 2009). Once activated, these cells can release a variety of neuroactive 97 substances, including proinflammatory cytokines (TNF, IL-1, IL-6), nitric oxide (NO), reactive oxygen species (ROS), arachidonic acid products, excitatory amino acids and ATP, increasing the excitability and reactivity of nociceptor neurons (Thameen et al., 2007; Faustino et al., 2011; Yamamoto et al., 2015).

Similarly, astrocyte resting and active states have been defined. It is classically considered that resting astrocytes perform a constant surveillance function, and express basal levels of GFAP (Gosselin et al., 2010). Nevertheless, it appears that initial microglial activation after injury can lead to astrocyte activation (Tanga et al., 2004). Activation of astrocytes generate morphological changes at the dorsal horn level, such as cellular hypertrophy and increased GFAP expression, leading to post-translational modifications that can increase the secretion of pro-inflammatory substances from spinal cord astrocytes and ultimately increase neuronal activity (Watkins \& Maier, 2005; Hansson, 2006). GFAP is the most widely used and a reliable marker for in vivo and in vitro identification of astrocytes (Tomassoni et al., 2004), and represents the major component of intermediate filaments in mature astroglia (Brenner, 1994). GFAP has been proven

111 to be important in modulating the motility and shape of astrocytes by giving structural stability to 112 the extensions of cell processes (Eng et al., 2000), in both normal and pathological brain 
113 (Bignami et al., 1972), and spinal cord tissues (Song et al., 2016). Despite its high sensitivity,

114 astrocyte labeling with GFAP antibody is usually accompanied by the detection of another

115 specific marker, called Connexin 43 (Cx-43) (Ochalsky et al., 1996), which is an important

116 component of astrocyte gap junctions (Rouach et al., 2002). Cx-43 maintains the normal shape

117 and function of astrocytes, which is important for their integrity and stability (Wu et al., 2015).

118 After peripheral or central nervous damage, Cx-43 expression markedly increases, and its

119 deletion in astrocytes can reduce acute astrogliosis, and can produce analgesia in different pain

120 models (Gao et al., 2010; Huang et al., 2012).

121 Microglia and astrocyte interaction has been shown to be involved in the establishment and

122 maintenance of pathological pain (Mika et al, 2013). Several studies suggest that microglia may

123 be temporarily involved in the initiation of pain, while astrocytic activation may be responsible

124 for its long-lasting maintenance (Gosselin et al., 2010). Although it is known that microglia and

125 astrocytes are potential therapeutic targets in pain control, no work has focused on the

126 description of these cells in horses under either normal conditions or during painful states. The

127 use of Iba-1, GFAP and Cx-43 in horses has been documented, mainly in encephalic diseases or

128 in embryotic/fetal development studies (Siso et al., 2003; Bielefeldt-Ohmann et al., 2017;

129 Rigoglio et al., 2017). However, an improved understanding of glial cell function and 130 morphology in healthy horses is needed to accurately know what happens in vitro and in vivo in

131 painful syndromes. The objective of this study was to determine healthy spinal microglia and

132 astrocyte morphology and distribution in equine spinal cord dorsal horns using Iba-1 and 133 GFAP/Cx-43 immunofluorescence labeling, respectively. 
136 MATERIAL AND METHODS

137 Animal selection and spinal cord sampling

138 The Ethics and Bioethic Committee for the Use of Animals in Research of the Universidad 139 Austral de Chile approved this project $\left(\mathrm{N}^{\circ} 001 / 2017\right)$. Five gelding mix breeds adult horses 140 (between 4 and 12 years of age) were selected from a commercial slaughterhouse (Nueva 141 Imperial Ltda., Temuco, Chile) and were evaluated in the holding pen, immediately prior to 142 humane euthanasia by pneumatic stunning and exsanguination, by an equine clinician. Animals 143 with visible wounds, gait alterations (either musculoskeletal or neurological) or other conditions 144 (pregnant, overweight, foal or senior horses) were excluded. Visual gait analysis was done by an 145 experienced equine clinician. Spinal cord tissues were collected immediately after euthanasia. 146 Lumbar spinal segments (L1-L6) were sampled and sectioned transversely maintaining the 147 integrity of the segments in their entire continuity and then stored in individual jars containing 148 Bouin fixative $(75 \mathrm{~mL}$ of saturated aqueous picric acid $(1.2 \% \mathrm{w} / \mathrm{v}), 25 \mathrm{~mL}$ of formalin $(40 \%$ $149 \mathrm{w} / \mathrm{v}$ formaldehyde), and $5 \mathrm{~mL}$ of glacial acetic acid), according to the method previously 150 described by Roales-Bujan et al., (2012). The cranial and caudal aspects of the spinal cord 151 segments were marked using $18 \mathrm{G}$ needles.

152

153

154

155

156

157

\section{Histology and immunofluorescence}

Spinal cord segments were fixed for 48 hours in Bouin fixative and then separated into $2 \mathrm{~mm}$ thick sections. Briefly, sections were first dehydrated with graded ethanol series $(70 \%$ to $100 \%$ for 1 hour each, plus an extra hour in $100 \%$ concentration), then with a mixture of $100 \%$ ethanol and pure butanol (1:1) for 1 hour, and finally a dehydration process with pure butanol (two sessions of 1 hour each) was performed. These segments were paraffin-embedded for 4 hours 
158 (divided into four separate sessions, 1 hour each at $60^{\circ} \mathrm{C}$ ), to be later sliced into $6 \mu \mathrm{m}$ transverse 159 sections using a manual rotatory microtome (Leica Biosystem RM2235, Germany). Each

160 histological section was dewaxed using pure xylene for 10 minutes each, rehydrated in alcohol 161 gradient (100\% to $70 \%, 5$ minutes each) and washed with distilled water. For epitope exposure 162 sections were treated with sodium citrate buffer (10mM Sodium Citrate, pH 6.0) and 163 microwaved three times for 4 minutes each $(700 \mathrm{~W})$. All sections were blocked for 1 hour with 164 TCT buffer $\left(0.25 \%\right.$ Casein, $0.15 \mathrm{M} \mathrm{NaCl}$, Triton X-100 0.5\% in TBS, pH 7.6) at $4^{\circ} \mathrm{C}$. Tissue 165 sections were then rinsed three times for 10 minutes in Tris-buffered saline (TBS) and incubated 166 with the primary antibody overnight at room temperature (RT). After incubation, tissue sections 167 were washed three times for 10 minutes in TBS and incubated with the corresponding secondary 168 antibody for 1 hour. Three spinal cord sections immediately adjacent were submitted for staining 169 with each biomarker antibody. A total of 15 spinal cord sections for each horse were used for 170 immunofluorescence analysis. For each biomarker, primary antibodies were omitted for negative 171 controls. Hematoxylin and eosin staining was performed in order to identify blood vessels, 172 neurons and check for tissue integrity.

Single immuno-labeling immunofluorescence

175 For immunodetection of spinal protoplasmic astrocytes, a polyclonal goat antibody raised against 176 Glial Fibrillary Acidic Protein (GFAP) (1:50, Santa Cruz Biotechnology, Santa Cruz, CA, USA)

177 and a polyclonal rabbit antibody against Connexin $43(\mathrm{Cx}-43)(1: 50$, Santa Cruz Biotechnology,

178 Santa Cruz, CA, USA) were used. For immunodetection of spinal microglia, a polyclonal rabbit 
179 antibody against a synthetic peptide corresponding to the C-terminus of ionized calcium-binding

180 adaptor molecule 1 (Iba-1) (1:50, Santa Cruz Biotechnology, Santa Cruz, CA, USA) was used.

181 Cx-43 and Iba-1 primary antibodies were incubated with an Alexa Fluor (labeled either with 488

182 or 594) (1:500, Invitrogen, Camarillo, CA, USA) conjugated goat anti-rabbit secondary antibody

183 for 1 hour at RT. Instead, GFAP primary antibody binding was visualized by incubation with

184 Alexa Fluor 488 conjugated donkey anti-goat secondary antibody (1:500, Invitrogen, Camarillo,

185 CA, USA) for 1 hour at RT. After immuno-labeling, sections were washed three times for 10

186 minutes in TBS and then counterstained with DAPI (1:5000, 4',6-diamidino-2-phenylindole,

187 dihydrochloride, Invitrogen, Camarillo, CA, USA) for 20 minutes at RT, washed with TBS and

188 mounted (Fluorescence Mounting Medium, DAKO).

\section{Double immuno-labeling immunofluorescence}

191

192

193

194

195

196

197

198

199

Co-localization assays were performed to detect spinal cord dorsal horn grey matter astrocytes and microglia (GFAP and Iba-1), and to confirm astrocyte labeling (GFAP and Cx-43). For double labeling with GFAP and Iba-1, tissue slides were initially processed as previously described and after blocking they were processed in the following order: they were first incubated overnight (day 1 ) with polyclonal goat primary antibody anti-GFAP (1:50, Santa Cruz Biotechnology, Santa Cruz, CA, USA), and were then incubated overnight (day 2) with polyclonal rabbit primary antibody anti-Iba-1 (1:50, Santa Cruz Biotechnology, Santa Cruz, CA, USA). The secondary antibodies were incubated sequentially, first with the conjugated anti-goat secondary antibody (Alexa Fluor 488, 1:500, Invitrogen) for 1 hour at RT. After three washes 
200 with TBS, the conjugated anti-rabbit secondary antibody (Alexa Fluor 594, 1:500, Invitrogen,

201 Camarillo, CA, USA) was incubated for another hour at RT. Finally, the slides were washed with

202 TBS, counterstained with DAPI (1:5000, Invitrogen, Camarillo, CA, USA) for 20 minutes at RT,

203 washed again with TBS and coverslip mounted. For double labeling with GFAP and Cx-43,

204 tissue slides were incubated with a polyclonal goat primary antibody anti-GFAP (1:50, Santa

205 Cruz Biotechnology, Santa Cruz, CA, USA) and a polyclonal rabbit primary antibody anti-Cx-43

206 (1:50, Santa Cruz Biotechnology, Santa Cruz, CA, USA). Finally, secondary antibodies were

207 incubated sequentially as described above (Alexa Fluor 488 conjugated anti-goat secondary

208 antibody and Alexa Fluor 594 conjugated anti-rabbit secondary antibody, and then

209 counterstained with DAPI).

210

211 Image collection and analysis

212 Iba-1, GFAP and Cx-43 immunoreactivity were analyzed to evaluate spinal astrocytes and

213 microglia morphology and organization. Immuno-labeled sections were visualized at 10X, 20X,

$21440 \mathrm{X}, 60 \mathrm{X}, 63 \mathrm{X}$ and 100X magnifications, and examined using an Eclipse E200 Biological

215 epifluorescence microscope and Olympus FV1000 confocal fluorescent microscope. Images

216 were captured with a Basler Scout scA780-541C camera and collected through Pylon Viewer 4

217 software for epifluorescence microscope images, and laser scanning was performed using

218 Olympus F10 Fluorview software for confocal microscope images. Z-stack of optical sections,

2190.5 to $0.9 \mu \mathrm{m}$ in total thickness, were captured from 15 spinal cord tissue slices from each horse

220 using a confocal microscope. At 10X magnification, an observation of the spinal cord was

221 performed to select those horses with spinal cord sections that had both dorsal horns completely

222 defined. Finally, five horses met all requirements and were included in the study. 
223 Morphologically, evaluation of shape, area (in spherical form cells) or cell diameter (in ramified

224 form cells), plus process length and thickness was performed using ImageJ. In the case of

225 microglial cell analysis, single cell immune-labeling with Iba-1 positive cells were considered;

226 for astrocytes, double cell immuno-labeling with GFAP (Alexa Fluor 488 labeled) and Cx-43

227 (Alexa Fluor 594 labeled) was implemented. The diameter of twenty ramified cells was

228 determined measuring the longest axis in non-overlapping cells, with a cross sectional line

229 through the nucleus (DAPI marked) and two endpoint branches. A line between the nucleus and

230 the end of a primary process was traced to measure the process length, and the thickness of these

231 processes was defined with a trace line between two parallel sides of the same process

232 (Supplementary File, A). For spherical form cells, cell surface was defined with the free hand

233 selection tool, and a line around twenty cell bodies (DAPI marked) was made to calculate the

234 cross sectional area of glial cells (Supplementary File, B).

235 To determine the number of microglial and astrocyte cells at the dorsal horn, the total number of

236 Iba-1, GFAP and Cx-43 labeled cells detected at 10X magnification in 15 dorsal horn sections

237 were counted. First, due to the large size of the horse spinal cord, several photos (2592 x 1944

238 pixels each) were taken to be later reconstructed using Adobe Illustrator CC 2015 software.

239 Using Image $\mathrm{J}$ software, cell counting was performed in a defined square perimeter of 1,000 $\mu \mathrm{m} 2$

240 (Supplementary File, C) in three different segments of the dorsal horn (30 random squares per

241 segment), defined as 1) Posterior dorsal horn (including lamina I, II and III), 2) Intermediate

242 dorsal horn (including lamina IV), and 3) Ventral dorsal horn (including lamina V and IV). All

243 data was collected in Microsoft Excel, and means and standard deviations of each previously

244 defined category were obtained. 


\section{RESULTS}

247 Morphology and organization of Iba-1 expressing microglia in horse spinal cord dorsal horns

248 Microglia showed a spherical or amoeboid shape (Fig. 1A to D), with a mean cell area of $92.4 \pm$

$24934 \mu \mathrm{m} 2$. Within the dorsal horn, Iba-1 fluorescence varied in all study subjects. At 10X

250 magnification, the distribution of Iba-1 microglial cells varied among areas within the dorsal

251 horn from the same horse. The higher number of Iba-1 positive cells were found in the posterior

252 dorsal horn, equally reactive in both dorsal horns (Fig. 1E). In all horses, the average number of

253 microglial cells was $9.4 \pm 1.8$ cells for the posterior dorsal horn, $3.8 \pm 3.1$ cells for the

254 intermediate dorsal horn, and $2.5 \pm 2.0$ cells for the ventral dorsal horn. Additionally, a close

255 association between microglia and astrocytes processes was observed using Iba-1 and GFAP

256 double immuno-labeling (Fig. 2B), mainly between microglial cell bodies and secondary

257 astrocyte branches.

Morphology and organization of GFAP and Cx-43 expressing protoplasmic astrocytes in horse

259

260

261

262

263

264

265

266

267 spinal cord dorsal horns

GFAP was mainly found in astrocytic primary processes in comparison to cell somas, highlighting a ramified cell shape (Fig. 2A). Also, most of the evaluated astrocytes had two to four primary processes, with a mean of two secondary processes arising from the primary. A close association between secondary processes and neighboring astrocytes and microglia (Fig. 2B), adjacent blood vessels (Fig. 2C) and neurons (Supplementary File 2) was observed. The arising secondary processes were only visualized using confocal microscopy. Double immunofluorescence with GFAP and Cx-43 was needed to better define astrocyte cell bodies and extensions of processes (Fig. 2D and E). According to GFAP and Cx-43 co-localization assays, 
268 horse astrocytes have an average diameter of $56 \pm 14 \mu \mathrm{m}$, with an average length for primary

269 processes of $28 \pm 8 \mu \mathrm{m}$, and an average thickness of $1.4 \pm 0.3 \mu \mathrm{m}$. Since it was not possible to

270 visualize the complete cell size at 10X magnification using only GFAP (soma and process could

271 not be defined at this magnification), the cellular density of astrocytes was defined using only

$272 \mathrm{Cx}-43$, which allowed us a better definition of the whole cell in comparison to GFAP. Astrocyte

273 cells were distributed throughout all laminae with a high, moderate and minimal fluorescence.

274 The average number of cells in each segment was: $6.1 \pm 2.0$ cells for the posterior dorsal horn,

$2753.6 \pm 1.2$ cells for the intermediate dorsal horn, and $1.6 \pm 1.0$ cell for the ventral dorsal horn.

276 Astrocytes were found in cell agglomerations along with other astrocytes, with minimal contact

277 between other groups and a tight connection between end-point processes and capillary walls

278 (Fig. 2F).

\section{DISCUSSION}

281 It is well known that microglia and astrocytes are potential therapeutic targets for pain control.

282 Therefore, it becomes necessary to understand how these cells maintain and modulate CNS

283 homeostasis (Old et al., 2015). Several studies have generated a list of glial-derived signaling

284 molecules and mediators involved in the neuron-glia interaction in acute and chronic states of

285 pain (Ji et al., 2013). Despite this, there is still a gap in our knowledge about how glial cells are

286 able to alter and maintain states of central sensitization, and even more, mask the analgesic

287 effects of opioids in chronic exposures (Watkins et al., 2007; Old et al., 2015). Until now, the

288 study and detection of these cells has been based on the use of immunomarkers, and of all of

289 them, Iba-1 and GFAP/Cx-43 have been essential to identify microglia and astrocytes,

290 respectively, using immunohistochemical, immunoblotting (Western blot analysis) and RT-PCR 
291 assays, in both clinical and basic neurobiological studies (Tetzlaff et al., 1988; Eng et al, 2000;

292 Tomassoni et al., 2004).

293 Spinal microglial cells in the dorsal horn of healthy horses

294 In this study, we demonstrated that Iba-1 can successfully immuno-label microglial cells in horse

295 spinal cords, and thus reveal microglia morphology and corresponding distribution within the

296 dorsal horn laminae of healthy horses. Horse microglial cells had an amoeboid and spherical

297 shape (Fig. 1A to D), mainly located in the same site as in rodents (Zhang et al., 2008), in the

298 superficial regions of the dorsal horn (Fig. 1E). Nevertheless, the conventional hyper-ramified

299 shape that is normally visible in resting microglial cells (Ito et al., 1998) was not found in any

300 subject. Typically, an amoeboid shape represents active or overactive states of microglial cells

301 after nerve damage (Eriksson et al., 1993). Regardless of this, recent studies have shown that

302 microglia's morphological characterization is more flexible and dynamic than previously

303 described, and microglial cell morphology can range from ramified cells to hypertrophied cells

304 with large somas, both in resting and active states (Zhang et al., 2008; Nimmerjahn et al., 2015).

305 A recent study reported that encephalitic diseases in horses induce an intense branching 306 appearance of microglial cells (Bielefeldt-Ohmann et al., 2017). Therefore, there is no evidence

307 that microglial cells in horses have a determined phenotype in both pathological and healthy

308 conditions. This information shows to be valuable when looking for the prototypic phenotype of

309 microglial cells in horses and should be considered. Apparently, the general concept that

310 different states of health can frame a specific cellular form does not exist in horses, just as it has

311 been proven in rodents, suggesting that more than one type of morphology may be found, and

312 thus, microglial morphology does not necessarily imply specific functionality. Nevertheless, both 
313 studies were performed using a small sample size, and we believe that a specific resting and

314 active microglial morphology cannot be defined.

317 GFAP has been extensively used as an astrocyte marker in several mammalian species in 318 addition to humans and rodents (Machado \& Alessi, 1997; Nielsen \& Jørgensen, 2003; Toda et 319 al., 2007; Sikasunge et al., 2009). However, there are no morphological nor structural 320 descriptions of astrocytes in equine spinal cord grey matter.

321 In our study, we demonstrated that GFAP and Cx-43 can immuno-label spinal astrocytes in 322 horses, and additionally could be used for study of the morphology and distribution of these 323 cells. In this study, a complete description of astrocytes' structure was complex for several 324 reasons. First, GFAP apparently was not detected in the whole cell; and it was mostly expressed 325 in primary processes, and to a much lesser extent in cellular bodies, as it has been described in 326 rodents (Bushong et al., 2002, Blechingberg et al., 2007). In order to better define astrocyte 327 structure, double immunolabeling of GFAP along with $\mathrm{Cx}-43$ was performed. This permitted a 328 better characterization of the morphology, dimension and cell density (Fig. 2D and E). 329 According to our findings, horse protoplasmic astroglia is smaller and structurally less complex 330 showing fewer processes compared to human astrocytes (Oberheim et al., 2009). Instead, horse 331 astrocytes show resemblance to those found in rodents. In terms of cell measurements, we 332 observed that horse astrocytes had an average diameter of $56.0 \pm 14 \mu \mathrm{m}$, with a primary process 333 length of $28.0 \pm 8 \mu \mathrm{m}$, and thickness of $1.4 \pm 0.3 \mu \mathrm{m}$. These same measurements in rodents were $33456.0 \pm 2.0 \mu \mathrm{m}, 37.2 \pm 2.0 \mu \mathrm{m}$, and $2.2 \pm 0.13 \mu \mathrm{m}$, respectively. Therefore, horse and rodent 
335

336

337 horses sit further away phylogenetically than rodents from humans, differences between horses

338 and humans could be affected by type of tissue sample and fixation methods. Therefore,

339 standardization of both factors must be controlled in future studies to determine these

340 morphological differences.

341 Similarly to microglia, the highest number of astrocytes was counted in the more superficial zone

342 of the dorsal horn (Ochalski et al., 1996). In rodents, this segment contains the main termination

343 zone of nociceptive primary afferents (Todd, 2010), therefore a correlation between

344 inflammatory/neuropathic conditions and a higher glial cells population could exist and

345 contribute in the maintenance of pain syndromes. Moreover, several studies in humans and

346 rodents explain that spinal astrocytes are organized into domains, where each cell occupies its

347 own anatomical space surrounding a neuronal synaptic space, with process projections that

348 penetrate the process-delimited domains of only a single neighboring astrocyte (Oberheim et al.,

349 2008). In this study, we showed some evidence that astrocytes located in the dorsal horn are

350 organized as well defined clusters around blood vessels (Fig. 2F). So far, there is accumulating

351 evidence that the neuron-glia-blood interaction at the spinal cord dorsal horn level is the basis for

352 the inter- and intra-cellular signaling mechanisms that influence synaptic activity, cell growth

353 and nutrition, in addition to the pain signaling that leads to exacerbate chronic and

354 neuropathological pain (Milligan \& Watkins, 2009; Sofroniew, 2009; Sofroniew \& Vinters,

355 2010). Nevertheless, more studies are needed to demonstrate the molecular mechanisms involved

356 in the neuron-glia interaction in horses. 
359 CONCLUSIONS

360 Although rodents have increased our understanding of pain mechanisms and have helped to 361 predict the effectiveness of certain analgesic mechanisms (Whiteside et al., 2008), our

362 knowledge of the molecular and cellular mechanisms that underlie chronic pain remains 363 substantially incomplete. Several authors have demonstrated that there are significant differences 364 in the metabolism and anatomy of rodents that can affect extrapolation of data to humans (Mogil 365 et al., 2010). Future research should focus on exploring other species that might reveal a glial 366 organization that is closer to human than rodents, in order to investigate the role of glial cells in 367 central sensitization and persistent pain in a more adequate temporal-spatial circumstance. The results described here, including shape, cell dimension and distribution within the dorsal horn of

369 microglia and astrocytes in healthy horses, characterize for the first time the morphology and organizational aspects of horse spinal glia. To our knowledge, this morphologic cell type has not been described before in healthy horse dorsal horns using immunofluorescence analysis. We believe that a clear understanding of the molecular mechanisms underlying horse glial function is necessary, and could represent a novel approach for efficient novel pharmacological targeting.

374 Upcoming studies will address the molecular and cellular modifications in the neuron-glia network under acute and chronic painful conditions in horses.

\section{ACKNOWLEDGEMENTS}

We gratefully thank Genaro Alvial (Research Assistant at Faculty of Medicine, Universidad

379 Austral de Chile) for his technical support in immunofluorescence analysis. 


\section{REFERENCES}

Bielefeldt-Ohmann H, Bosco-Lauth A, Hartwig AE, Uddin MJ, Barcelon J, Suen WW, Wang W, Hall RA, Bowen RA. 2017. Characterization of non-lethal West Nile Virus (WNV) infection in horses: Subclinical pathology and innate immune response. Microbial Pathogenesis 103: 71-79.

Bignami A, Eng LF, Dahl D, Uyeda CT. 1972. Localization of the glial fibrillary acidic protein 387 in astrocytes by immunofluorescence. Brain Research 43: 429-435.

Blechingberg J, Holm IE, Nielsen KB, Jensen TH, Jorgensen AL, Nielsen AL. 2007. Identification and characterization of GFAPkappa, a novel glial fibrillary acidic protein isoform. Glia 55: 497-507.

Brenner M. 1994. Structure and transcriptional regulation of the GFAP gene. Brain Pathology 4: 245-257.

Bushong EA, Martone MA, Jones YZ, Ellisman MH. 2002. Protoplasmic astrocytes in CA1 atratum radiatum occupy separate anatomical domains. Journal of Neuroscience 22: 183-192. hemichannels and gap junctions play a crucial role in development of chronic neuropathic pain

397 following spinal cord injury. Glia 60: 1660-1670. (1969-2000). Neurochemical Research 25: 1439-1451. 
400 Eriksson NP, Persson JK, Svensson M, Arvidsson J, Molander C, Aldskogius H. 1993. A 401 quantitative analysis of the microglial cell reaction in central primary sensory projection 402 territories following peripheral nerve injury in the adult rat. Experimental Brain Research 96: 1940327.

404 Faustino JV, Wang X, Johnson CE, Klibanov A, Derugin N, Wendland MF, Vexler ZS. 2011. 405 Microglial cells contribute to endogenous brain defenses after acute neonatal focal stroke. 406 Journal of Neuroscience Research 31: 12992-13001.

407 Gao YJ, Ji RR. 2010. Targeting astrocyte signaling for chronic pain. Neurotherapeutics 7: 482408493.

409 Gosselin RD, Suter M, Ji RR, Decosterd I. 2010. Glial cells and chronic pain. Neuroscientist 16: $410 \quad 519-531$.

411 Hains BC, Waxman SG. 2006. Activated microglia contribute to the maintenance of chronic pain 412 after spinal cord injury. Journal of Neuroscience 26: 4308-4317.

413 Hald A, Nedergaard S, Hansen RR, Ding M, Heegaard AM. 2009. Differential activation of 414 spinal cord glial cells in murine models of neuropathic and cancer pain. European Journal of Pain $415 \quad 13: 138-145$.

416 Hanisch UK, Kettenmann H. 2007. Microglia: active sensor and versatile effector cells in the 417 normal and pathologic brain. Nature Neuroscience 10: 1387-1394.

418 Hansson E. 2006. Could chronic pain and spread of pain sensation be induced and maintained by 419 glial activation? Acta Physiologica 187: 321-327. 
420 Haydon PG. 2001. Glia: listening and talking to the synapse. Nature Reviews Neuroscience 2: $421 \quad 185-193$.

422 Huang C, Han X, Li X, Lam E, Peng W, Lou N, Torres A, Yang M, Garre JM, Tian GF, Bennett 423 M, Nedergaard M, Takano T. 2012. Critical role of connexin 43 in secondary expansion of 424 traumatic spinal cord injury. Journal of Neuroscience 32: 3333-3338.

425 Ito D, Imai Y, Ohsawa K, Nakajima K, Fukuuchi Y, Kohsaka S. 1998. Microglia-specific 426 localisation of a novel calcium binding protein, Iba1. Molecular Brain Research 57: 1-9.

427 Ji RR, Berta T, Nedergaard M. 2013. Glia and pain: is chronic pain a gliopathy? Pain 154: 10-28.

428 Machado GF, Alessi AC. 1997. Glial fibrillary acidic protein (GFAP) immunoreactive astrocytes 429 in the CNS of normal and rabies-infected adult cattle. I. Hippocampus and dentate gyrus. 430 Brazilian Journal of Veterinary Research and Animal Science 34: 345-348.

431 Mika J, Zychowska M, Popiolek-Barczyk K, Rojewska E, Przewlocka B. 2013. Importance of 432 glial activation in neuropathic pain. European Journal of Pharmacology 716: 106-119.

433 Milligan ED, Watkins LR. 2009. Pathological and protective roles of glia in chronic pain. Nature 434 Reviews Neuroscience 10: 23-36.

435 Mogil JS, Davis KD, Derbyshire SW. 2010. The necessity of animal models in pain research. 436 Pain 151: 12-17.

437 Nielsen AL, Jørgensen AL. 2003. Structural and functional characterization of the zebrafish gene 438 for glial fibrillary acidic protein, GFAP. Gene 310: 123-132.

439 Nimmerjahn A, Kirchhoff F, Helmchen F. 2005. Resting microglial cells are highly dynamic 440 surveillants of brain parenchyma in vivo. Science 308: 1314-1318. 
441 Oberheim NA, Takano T, Han X, He W, Lin JH, Wang F, Xu Q, Wyatt JD, Pilcher W, Ojemann

442 JG, Ransom BR, Goldman SA, Nedergaard M. 2009. Uniquely hominid features of adult human 443 astrocytes. Journal of Neuroscience 29: 3276-3287.

444 Oberheim NA, Tian GF, Han X, Peng W, Takano T, Ransom B, Nedergaard M. 2008. Loss of 445 astrocytic domain organization in the epileptic brain. Journal of Neuroscience 28: 3264-3276.

446 Ochalski P, Frankenstein UN, Hertzberg EL, Nagy JI. 1996. Connexin-43 in rat spinal cord: 447 localization in astrocytes and identification of heterotypic astro-oligodendrocytic gap junctions. 448 Neuroscience 76: 931-945.

449 Old EA, Clark AK, Malcangio M. 2015. The role of glia in the spinal cord in neuropathic and 450 inflammatory pain. In: Schaible HG, ed. Pain Control. Berlin: Springer Berlin Heidelberg, 145451170.

452 Rigoglio NN, Barreto RS, Favaron PO, Jacob JC, Smith LC, Gastal MO, Gastal E, Miglino MA. 453 2017. Central Nervous System and Vertebrae Development in Horses: A Chronological Study 454 with Differential Temporal Expression of Nestin and GFAP. Journal of Molecular Neuroscience 455 61: 61-78.

456 Roales-Buján R, Páez P, Guerra M, Rodríguez S, Vío K, Ho-Plagaro A, García-Bonilla M, 457 Rodríguez-Pérez LM, MD Domínguez-Pinos, Rodríguez EM, Pérez-Fígares JM. 2012. 458 Astrocytes acquire morphological and functional characteristics of ependymal cells following 459 disruption of ependyma in hydrocephalus. Acta neuropathologica, 124: 531-546.

460 Rouach N, Avignone E, Même W, Koulakoff A, Venance L, Blomstrand F, Giaume C. 2002. 461 Gap junctions and connexin expression in the normal and pathological central nervous system. 462 Biologie Cellulaire 94: 457-475. 
463 Sikasunge CS, Johansen MV, Phiri IK, Willingham AL, Leifsson PS. 2009. The immune 464 response in Taenia solium neurocysticercosis in pigs is associated with astrogliosis, axonal

465 degeneration and altered blood-brain barrier permeability. Veterinary Parasitology 160: 242466250.

467 Siso S, Ferrer I, Pumarola M. 2003. Abnormal synaptic protein expression in two Arabian horses 468 with equine degenerative myeloencephalopathy. Veterinary Journal 166: 238-243.

469 Sofroniew MV, Vinters HV. 2010. Astrocytes: biology and pathology. Acta Neuropathologica 470 119: 7-35.

471 Sofroniew MV. 2009. Molecular dissection of reactive astrogliosis and glial scar formation. 472 Trends in Neurosciences 32: 638-647.

473 Song ZP, Xiong BR, Guan XH, Cao F, Manyande A, Zhoun Y, Xheng H, Tian Y. 2016. 474 Minocycline attenuates bone cancer pain in rats by inhibiting NF-kB in spinal astrocytes. Acta 475 Pharmacologica Sinica 37: 753-762.

476 Tanga FY, Raghavendra V, DeLeo JA. 2004. Quantitative realtime RT-PCR assessment of spinal 477 microglial and astrocytic activation markers in a rat model of neuropathic pain. Neurochemistry 478 International 45: 397-407.

479 Temburni MK, Jacob MH. 2001. New functions for glia in the brain. Proceedings of the National 480 Academy of Sciences 98: 3631-3632.

481 Tetzlaff W, Graeber MB, Bisby MA, Kreutzberg GW. 1988. Increased glial fibrillary acidic 482 protein synthesis in astrocytes during retrograde reaction of the rat facial nucleus. Glia 1: 90-95. 
483 Thameem DS, Kaur C, Ling EA. 2007. Microglial activation and its implications in the brain 484 diseases. Current Medicinal Chemistry 14: 1189-1197.

485 Toda Y, Matsuki N, Shibuya M, Fujioka I, Tamahara S, Ono K. 2007. Glial fibrillary acidic 486 protein (GFAP) and anti-GFAP autoantibody in canine necrotising meningoencephalitis. 487 Veterinary Record 161: 261-264.

488 Todd AJ. 2010. Neuronal circuitry for pain processing in the dorsal horn. Nature reviews. 489 Neuroscience 11: 823-853.

490 Tomassoni D, Avola R, Di Tullio MA, Sabbatini M, Vitaioli L, Amenta F. 2004. Increased 491 expression of glial fibrillary acidic protein in the brain of spontaneously hypertensive rats. 492 Clinical and Experimental Hypertension 26: 335-350.

493 Watkins LR, Hutchinson MR, Ledeboer A, Wieseler-Frank J, Milligan ED, Maier SF. 2007. Glia 494 as the "bad guys": implications for improving clinical pain control and the clínical utility of 495 opioids. Brain Behavior and Immunity 21: 131-146.

Watkins LR, Maier SF. 2005. Immune regulation of central nervous system functions: from 497 sickness responses to pathological pain. Journal of Internal Medicine 257: 139-155.

498 Watkins LR, Milligan ED, Maier SF. 2005. Glial proinflammatory cytokines mediate 499 exaggerated pain states: implications for clinical pain. Advances in Experimental Medicine and 500 Biology 521: 1-21.

501 Whiteside GT, Adedoyin A, Leventhal L. 2008. Predictive validity of animal pain models? A 502 comparison of the pharmacokinetic-pharmacodynamic relationship for pain drugs in rats and 503 humans. Neuropharmacology 54: 767-775. 
$504 \mathrm{Wu}$ LY, Yu XL, Feng LY. 2015. Connexin 43 stabilizes astrocytes in a stroke-like milieu to 505 facilitate neuronal recovery. Acta Pharmacologica Sinica 36: 928-938.

506 Yamamoto Y, Terayama R, Kishimoto N, Maruhama K, Mizutani M, Iida S, Sugimoto T. 2015. 507 Activated microglia contribute to convergent nociceptive inputs to spinal dorsal horn neurons 508 and the development of neuropathic pain. Neurochemical Research 40: 1000-1012.

509 Zhang F, Vadakkan KI, Kim SS, Wu LJ, Shang Y, Zhuo M. 2008. Selective activation of 510 microglia in spinal cord but not higher cortical regions following nerve injury in adult mouse.

511 Molecular Pain 4: 15-31.

512 


\section{Figure 1}

Microglial immunostaining against Iba-1 in equine spinal cord dorsal horns (Iba-1, green; DAPI, blue).

Microglia showed a spherical or amoeboid shape, constantly throughout the dorsal horn (63X magnification, epifluorescence microscopy) (A, B, C and D). Distribution of Iba-1 marked microglia varied in different areas of the dorsal horn, with a higher microglial cell population in the posterior dorsal horn (i.e. laminae I, II and III) (10X magnification, epifluorescence microscopy) (E). Scale bar, $20 \mu \mathrm{m}(n=5)$.
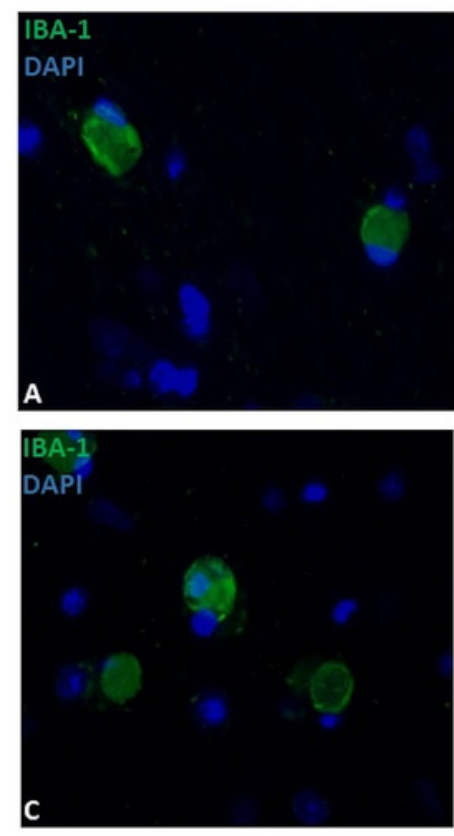
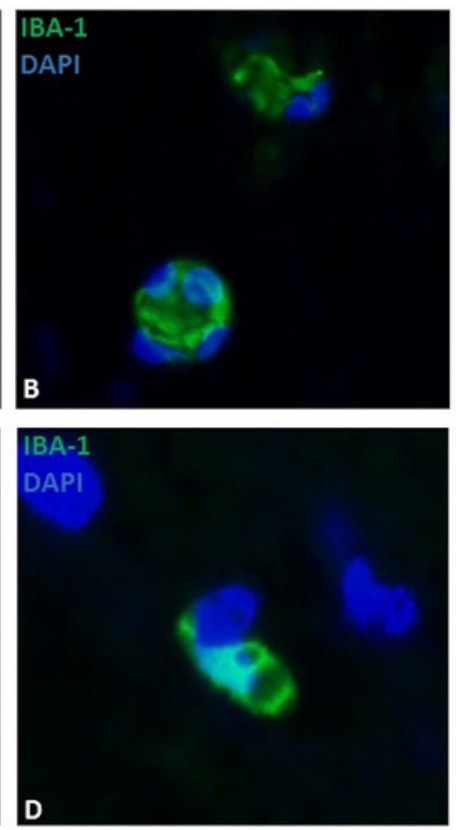

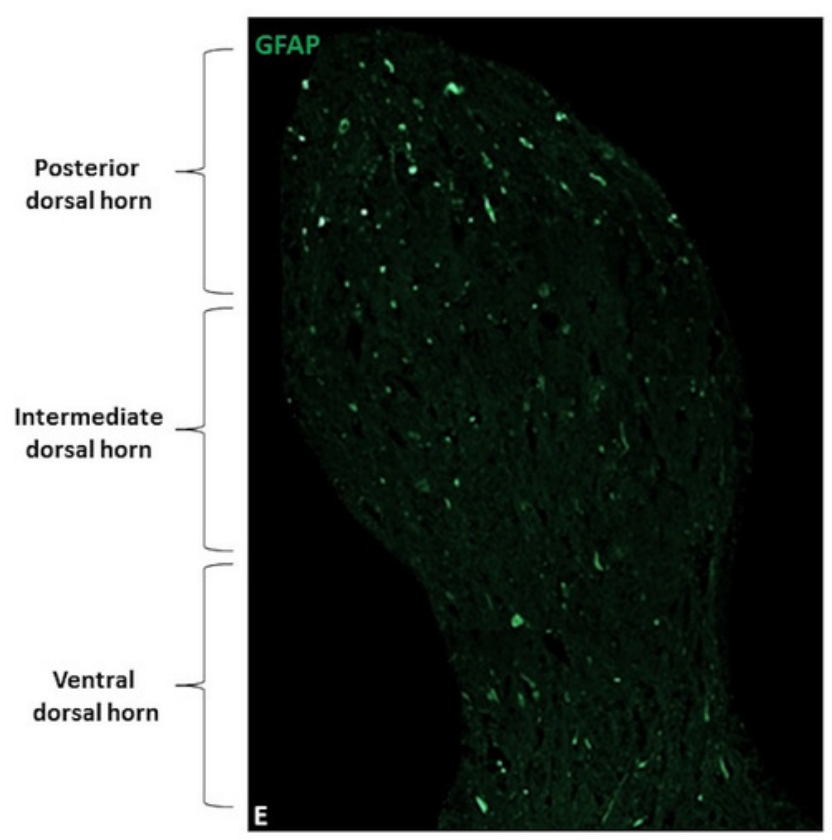




\section{Figure 2}

Representative epifluorescence and confocal images of longitudinal sections of spinal cord dorsal horn in healthy horses.

The sections were double immune-labeled against GFAP (green), Cx-43 (red) and DAPI (blue) to detect astrocytes, and double immune-labeled against GFAP (green), Iba-1 (red) and DAPI (blue) to detect astrocytes and microglial cells. Astrocytes have increased GFAP expression towards the end of their primary processes, and the thin secondary processes arising from the primary processes were in close contact with neighboring astrocytes and microglia (B), vessels ( $C$; bv) and neurons (Supplementary File 2). Cx-43 was located in cell bodies (D) and processes $(E)$, and astrocytes were found in cell agglomerations along with other astrocytes (F). Images at $60 \mathrm{X}$ magnification, scale bar $20 \mu \mathrm{m}(\mathrm{n}=5)$. 

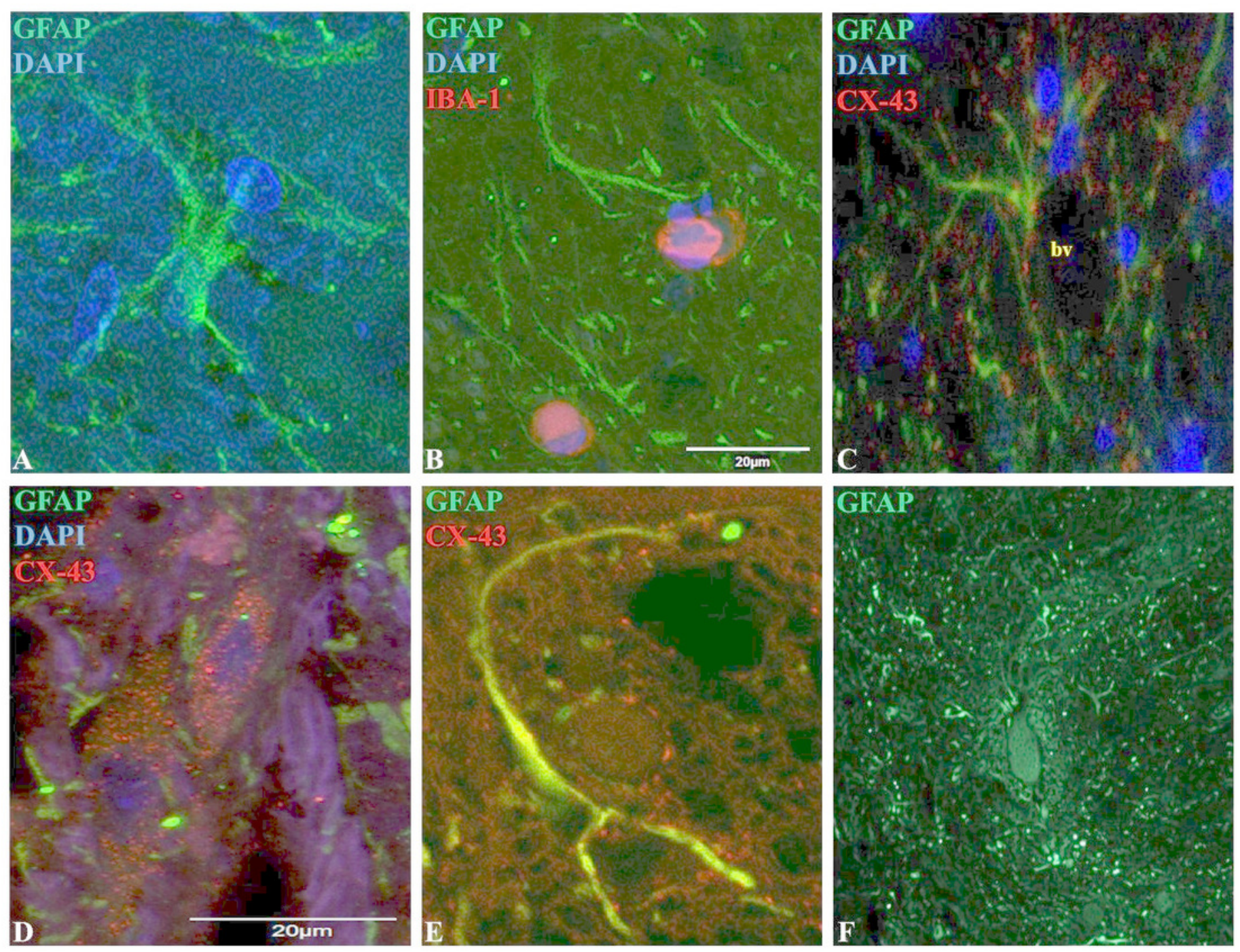